\title{
Cesarean delivery is not associated with decreased at-birth fracture rates in osteogenesis imperfecta
}

\author{
S. Bellur, BA 1 , M. Jain, MD, PhD ${ }^{1}$, D. Cuthbertson, MS², D. Krakow, MD ${ }^{3-5}$, J.R. Shapiro, MD ${ }^{6}$, \\ R.D. Steiner, $\mathrm{MD}^{7-9}$, P.A. Smith, $\mathrm{MD}^{10}$, M.B. Bober, MD ${ }^{11}$, T. Hart, BS ${ }^{12}$, J. Krischer, $\mathrm{PhD}^{2}$, \\ M. Mullins, BSN ${ }^{1}$, P.H. Byers, MD ${ }^{13,14}$, M. Pepin, MS, LGC ${ }^{13,14}$, M. Durigova, PhD ${ }^{15}$, \\ F.H. Glorieux, MD, PhD ${ }^{15}$, F. Rauch, MD ${ }^{15}$, V.R. Sutton, $\mathrm{MD}^{1,16}$, B. Lee, MD, PhD ${ }^{1,16}$, \\ Members of the BBD Consortium and S.C. Nagamani, MD ${ }^{1,16}$
}

\begin{abstract}
Purpose: Osteogenesis imperfecta (OI) predisposes to recurrent fractures. Patients with the moderate to severe forms of OI present with antenatal fractures, and the mode of delivery that would be safest for the fetus is not known.

Methods: We conducted systematic analyses of the largest cohort of individuals with OI $(n=540)$ enrolled to date in the OI Linked Clinical Research Centers. Self-reported at-birth fracture rates were compared among individuals with OI types I, III, and IV. Multivariate analyses utilizing backward-elimination logistic regression model building were performed to assess the effect of multiple covariates, including method of delivery, on fracture-related outcomes.
\end{abstract}

Results: When accounting for other covariates, at-birth fracture rates did not differ based on whether delivery was by vaginal route or by cesarean delivery (CD). Increased birth weight conferred higher risk for fractures irrespective of the delivery method. In utero fracture, maternal history of OI, and breech presentation were strong predictors for choosing $\mathrm{CD}$.

Conclusion: Our study, the largest to analyze the effect of various factors on at-birth fracture rates in OI, shows that CD is not associated with decreased fracture rate. With the limitation that the fracture data were self-reported in this cohort, these results suggest that $\mathrm{CD}$ should be performed only for other maternal or fetal indications, not for the sole purpose of fracture prevention in OI.

Genet Med advance online publication 1 October 2015

Key Words: at-birth fracture; cesarean delivery; in utero fracture; natural history study; osteogenesis imperfecta

\section{INTRODUCTION}

Osteogenesis imperfecta (OI), a genetically heterogeneous connective-tissue disorder, is the most common skeletal dysplasia predisposing to significantly increased bone fragility and fractures. ${ }^{1}$ Whereas the classification of OI has undergone a significant change with the discovery of new genes implicated in OI, the vast majority of patients (8590\%) can be categorized into types I, II, III, and IV that were originally proposed by Sillence. ${ }^{2-4}$ Type I is the mildest form and type II the most severe and perinatally lethal form, whereas types III and IV are moderate to severe forms of the disorder.

The widespread use of ultrasound during pregnancy and the availability of genetic testing have increased the antenatal diagnosis of OI. ${ }^{5-7}$ Most fetuses with moderate to severe OI and some with the mild forms sustain in utero fractures, and a valid clinical concern is to minimize trauma during delivery. Cesarean delivery $(\mathrm{CD})$ has been suggested as a more favorable delivery method by some based on the notion that this method is "more controlled" and "less traumatic" than vaginal delivery and thus could potentially decrease mortality and morbidity. ${ }^{8-10}$ However, there is no evidence that $\mathrm{CD}$ is superior to vaginal delivery. The only study to address at-birth fracture rates based on mode of delivery found that CD neither decreased fracture rates among those with the nonlethal forms nor prolonged survival among those with lethal forms of OI; however, limitations in sample size precluded a robust analysis of covariates related to fracture outcomes. ${ }^{11}$

\footnotetext{
Members of the Brittle Bone Disease Consortium are listed in the acknowledgements.

The first two authors contributed equally to this work.

${ }^{1}$ Department of Molecular and Human Genetics, Baylor College of Medicine, Houston, Texas, USA; ${ }^{2}$ College of Medicine, University of South Florida, Tampa, Florida, USA;

${ }^{3}$ Department of Orthopedic Surgery, University of California, Los Angeles, California, USA; ${ }^{4}$ Department of Human Genetics, University of California, Los Angeles, California, USA; ${ }^{5}$ Department of Obstetrics and Gynecology, University of California, Los Angeles, California, USA; ${ }^{6}$ Department of Bone and Osteogenesis Imperfecta, Kennedy Krieger Institute, Baltimore, Maryland, USA; ${ }^{7}$ Department of Pediatrics, Oregon Health \& Science University, Portland, Oregon, USA; ${ }^{8}$ Department of Molecular and Medical Genetics, Oregon Health \& Science University, Portland, Oregon, USA; ${ }^{9}$ Marshfield Clinic Research Foundation and University of Wisconsin, Marshfield and Madison, Wisconsin, USA; ${ }^{\circ}$ Shriners Hospitals for Children, Chicago, Illinois, USA; ${ }^{11}$ Division of Medical Genetics, Alfred I. DuPont Hospital for Children, Wilmington, Delaware, USA; ${ }^{12}$ Osteogenesis Imperfecta Foundation, Gaithersburg, Maryland, USA; ${ }^{13}$ Department of Medicine, Division of Medical Genetics, University of Washington, Seattle, Washington, USA; ${ }^{14}$ Department of Pathology, Division of Medical Genetics, University of Washington, Seattle, Washington, USA; ${ }^{15}$ Department of Orthopedic Surgery, Shriners Hospital for Children and McGill University, Montreal, Québec, Canada; ${ }^{16}$ Texas Children’s Hospital, Houston, Texas, USA. Correspondence: Sandesh C.S. Nagamani (Nagamani@bcm.edu)
}

Submitted 11 February 2015; accepted 14 August 2015; advance online publication 1 October 2015. doi:10.1038/gim.2015.131 
Providing evidence-based answers to clinically relevant questions in OI has been made difficult by the rarity of the condition. To advance clinical research and improve the care of patients with OI, the Linked Clinical Research Centers (LCRC), a network of five clinical centers with significant experience in treating patients with OI, was established in $2009 .{ }^{3}$ Using data from 540 individuals with OI who were enrolled in the Longitudinal Study of Osteogenesis Imperfecta conducted by the LCRC, we systematically analyzed the effects of various factors, including mode of delivery, on the at-birth fracture rates in OI. This study is the largest to date to address whether CD has an effect on at-birth fracture rates and whether an antenatal diagnosis of $\mathrm{OI}$ influences the choice of delivery method. In addition, we attempt to define novel correlations for fractures associated with birth trauma.

\section{Study population}

\section{MATERIALS AND METHODS}

The establishment of the LCRC and the subjects enrolled in the Longitudinal Study of Osteogenesis Imperfecta have been previously described. ${ }^{3}$ The LCRC comprised five clinical sites across North America: Baylor College of Medicine (Houston, TX); Kennedy Krieger Institute (Baltimore, MD) in collaboration with Nemours/Alfred I. DuPont Hospital for Children (Wilmington, DE); Oregon Health \& Science University (Portland, OR); and Shriners Hospital for Children (Chicago, IL, and Montreal, Canada). The Data Collection and Analysis Center at the University of South Florida College of Medicine (Tampa, FL) served as the central facility for data collection and analyses. The Collagen Diagnostic Laboratory at the University of Washington (Seattle, WA) served as the center for molecular and biochemical analysis. The institutional review boards of the participating sites approved the research protocol. Informed consent was obtained from subjects or their legal guardians.

A total of 540 individuals with OI were enrolled in the longitudinal study based on a previously published protocol. ${ }^{3}$ Of these

Table 1 Characteristics of patients with nonlethal, type I collagen-related osteogenesis imperfecta enrolled in the Linked Clinical Research Centers

\begin{tabular}{lccc} 
& OI type I & OI type III & OI type IV \\
\hline Total patients, $n$ & 239 & 101 & 147 \\
Sex, $n(\%)$ & & & \\
$\quad$ Male & $113(47.3)$ & $43(42.6)$ & $64(43.5)$ \\
$\quad$ Female & $126(52.7)$ & $58(57.4)$ & $83(56.5)$ \\
\hline Family history present, $n(\%)$ & $148(61.9)$ & $7(6.93)$ & $42(28.6)$ \\
\hline In utero fractures present, $n(\%)$ & $14(5.85)$ & $65(64.4)$ & $52(35.4)$ \\
\hline $\begin{array}{l}\text { Prenatal diagnosis, } n(\%) \\
\text { Presentation at birth, }{ }^{a} n\end{array}$ & $78(32.6)$ & $38(37.6)$ & $45(30.6)$ \\
$\quad$ Vertex & & & \\
$\quad$ Breech & 189 & 50 & 101 \\
\hline
\end{tabular}

Percentages in parentheses depict the proportion within each osteogenesis imperfecta (OI) subtype.

anformation about presentation at birth was unavailable for 73 individuals. individuals, 239 had OI type I, 147 had OI type IV, and 101 had OI type III $(n=487)$ (Table 1$)$ and were included in further analyses. The numbers of individuals with other subtypes of OI were very small (OI type V, $n=16$; OI type VI, $n=12$; OI type VII, $n=6$; unclassified OI, $n=19$ ) and hence were excluded from this study. Individuals with OI type II also were excluded because of the severity of the phenotype. Classification was predominantly based on clinical features; however, genotypic information was used to reclassify patients whenever available. Ten subjects originally classified as OI types III or IV were reclassified as types V, VII, or the "unclassified" categories based on genotype.

The data collected for this study included gender, age at enrollment, antenatal diagnosis (yes or no), presentation at birth (breech or vertex), family history (yes or no), family members with OI (mother, father, or sibling), in utero fractures (yes or no), number of fractures at birth, specific locations of at-birth fractures (long bone, rib, clavicle, and/or skull), gestational age at delivery, birth weight, birth length, and mode of delivery. Mode of delivery was categorized as vaginal (spontaneous or induced) or CD. A total of 454 subjects had data for all the covariates and were included in the primary analyses. The data were collected in an identical fashion at every site using online case report forms in accordance with the instructions outlined in the Manual of Operations. The Data Collection and Analysis Center monitored data delinquency and sent queries to centers to further improve the quality of the data.

\section{Statistical analysis}

Differences in the demographic characteristics were assessed using the chi-square test. The Mann-Whitney $U$-test was used to determine the differences between median birth weight and length. The proportions of individuals with at-birth fracture based on OI subtype and delivery method (vaginal versus CD) were analyzed using the chi-square test $(n=454)$; the Fisher exact test was applied when the numbers were less than five in any cell. To identify whether mode of delivery had an effect on fracture rates at birth, we further examined the data using logistic regression. We applied a backward-elimination strategy whereby all variables were included in a full model and, at each iteration, an independent variable that did not improve the model at the $P=0.05$ level using the likelihood ratio chisquared test was removed. Backward elimination was stopped when all included variables improved the model. In the model, birth fracture was included as a binary dependent variable. Independent variables that were included include delivery method (CD or vaginal delivery), OI subtype (type I, III, or IV), presence of in utero fractures (yes or no), gender (male or female), family history of OI (yes or no), family members with OI (mother, father, or sibling), antenatal diagnosis of OI (yes or no), presentation (breech or vertex), gestational age (in weeks), birth weight, and birth length. Interaction terms were not included in the full model because of concerns for overfitting given the large number of potential interactions. For variables that remained in the model after backward elimination, interaction terms were evaluated. The interaction between that 
variable with an independent effect and each other variable, stated above, was added and evaluated for model improvement by the likelihood ratio chi-squared test, with a threshold of $P=0.05$ for inclusion. We also examined the interaction between antenatal diagnosis and delivery method.

To analyze whether certain characteristics influenced the decision to choose a particular delivery method, we used logistic regression with a backward-elimination strategy to identify novel correlations, where mode of delivery was the binary dependent variable. Covariates considered included in utero fractures, maternal history of OI, and presentation at birth. Interaction terms were also evaluated to discern whether each variable independently correlated with the outcome measure.

\section{Study population}

\section{RESULTS}

The analysis of demographic characteristics showed that the proportions of individuals with each subtype in the study were generally representative of the prevalence observed in the broader population ${ }^{3,12}$ (Table 1). Patients with nonlethal dominant forms of type I collagen-related OI (i.e., types I, III, and IV) accounted for $90 \%$ of all subjects. Family history of OI was present in $62 \%$ of those with OI type I, whereas only $29 \%$ with OI type IV and $7 \%$ with OI type III had a parent with OI $(P<0.001)$. As expected, in utero fracture rates were highest among those with OI type III and lowest among those with OI type I; they differed significantly between the three subtypes $(P<0.001)$. The frequency of antenatal diagnosis in the various subtypes ranged from 31 to $38 \%$, and there were no intergroup differences; this is most likely because the majority of subjects with type I OI also had a family history of the condition, thus increasing the rate of detection of this milder form, which is otherwise difficult to diagnose antenatally.

The proportion of preterm deliveries of fetuses with the three subtypes of OI was not different $(0.13,0.16$, and 0.12 for OI types I, III, and IV, respectively), and the majority of deliveries occurred at full-term gestation (37-42 weeks). The prevalence of breech presentation was highest among those with OI type III, followed by those with type IV and I $(39.2,18.6$, and $8.6 \%$, respectively; $P<0.001)$. The likelihood of having a breech presentation at term in OI type III was 6.93 (95\% confidence interval (CI) 3.61-13.3; $P<0.001$ ) and 2.39 (CI: 1.23-4.64; $P=0.002)$ when compared with types I and IV, respectively.
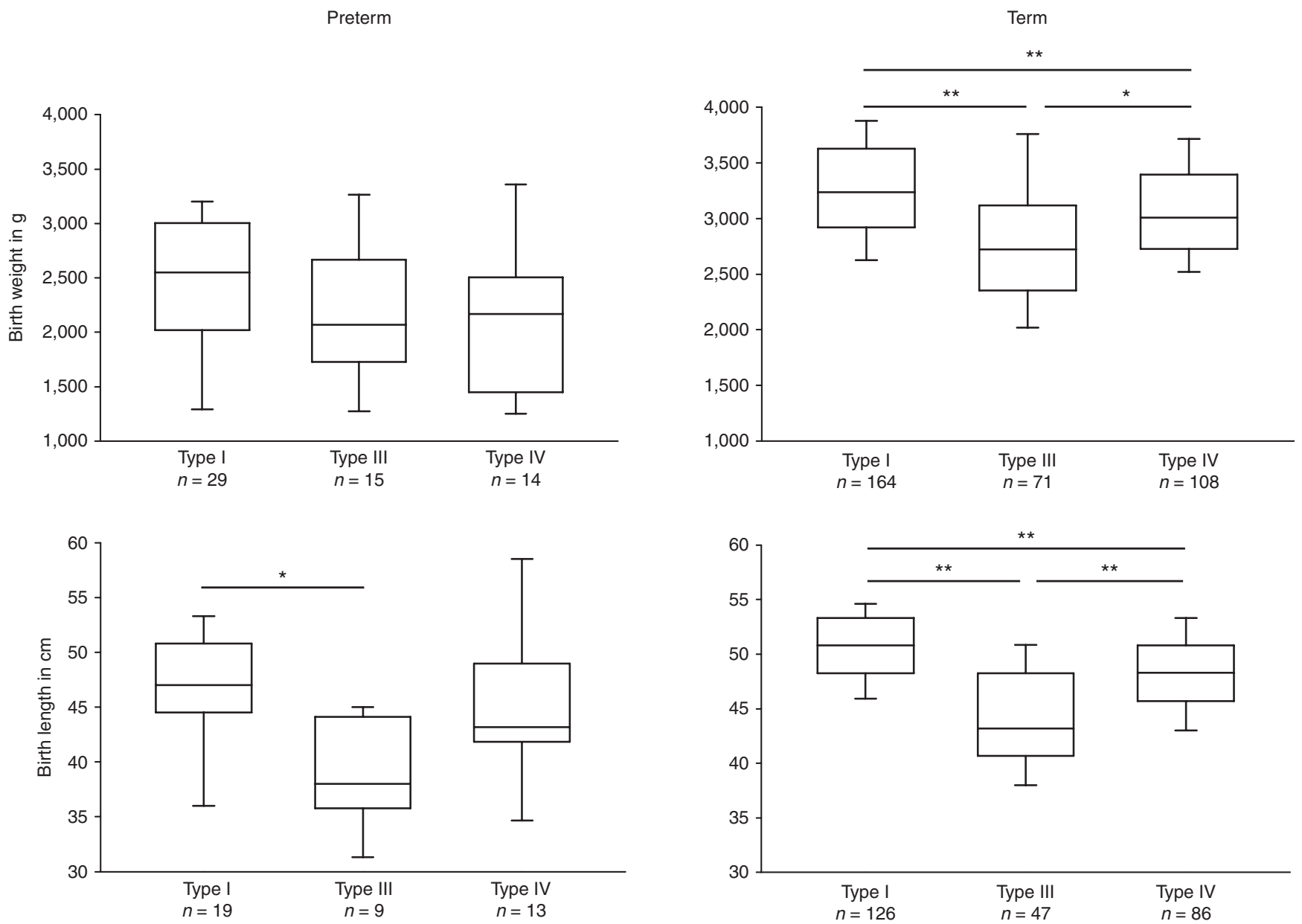

Figure 1 Birth weight and length of subjects with osteogenesis imperfecta (OI). Box plots of the birth weight and length of subjects with OI, depicting the median and 25 th and 75 th centiles. The error bars represent the 5th and 95th centiles. Preterm gestational age <37 weeks; term gestation $37-42$ weeks. ${ }^{*} P<0.01,{ }^{*} P<0.001$, Mann-Whitney $U$-test). 
Table 2 At-birth fracture rates based on osteogenesis imperfecta subtype and mode of delivery

\begin{tabular}{|c|c|c|c|c|c|}
\hline Ol type & $\begin{array}{l}\text { Delivery } \\
\text { type }\end{array}$ & $\begin{array}{c}\text { Patients } \\
\text { with fractures }(n)\end{array}$ & $\begin{array}{l}\text { Patients with } \\
\text { no fractures } \\
\text { (n) }\end{array}$ & $\begin{array}{c}\text { Proportion with } \\
\text { fractures }\end{array}$ & $\begin{array}{c}P \\
\text { value }^{\mathrm{a}}\end{array}$ \\
\hline \multirow[t]{2}{*}{1} & VD & 25 & 130 & 0.161 & \\
\hline & Total & 38 & 183 & 0.172 & \\
\hline III & VD & 39 & 4 & 0.907 & \\
\hline \multirow[t]{3}{*}{ IV } & VD & 42 & 35 & 0.545 & \\
\hline & $C D$ & 28 & 33 & 0.459 & 0.313 \\
\hline & Total & 70 & 68 & 0.507 & \\
\hline \multirow[t]{2}{*}{ All Types } & VD & 106 & 169 & 0.385 & \\
\hline & $C D$ & 90 & 89 & 0.503 & 0.014 \\
\hline
\end{tabular}

aDenotes $\alpha$ value for comparison between vaginal delivery (VD) and cesarean delivery (CD) within each osteogenesis imperfecta (OI) subtype.

Table 3 At-birth fracture rates in antenatally vs. postnatally diagnosed osteogenesis imperfecta

\begin{tabular}{lcccc}
$\begin{array}{l}\text { Delivery by } \\
\text { type of OI }\end{array}$ & $\begin{array}{c}\text { Patients } \\
\text { with } \\
\text { fractures }(\boldsymbol{n})\end{array}$ & $\begin{array}{c}\text { Patients } \\
\text { with no } \\
\text { fractures }(\boldsymbol{n})\end{array}$ & $\begin{array}{c}\text { Proportion } \\
\text { with } \\
\text { fracture }\end{array}$ & $\begin{array}{c}\boldsymbol{P} \\
\text { value }^{\text {a }}\end{array}$ \\
\hline $\begin{array}{l}\text { Antenatally } \\
\text { diagnosed OI }\end{array}$ & & & & 0.011 \\
VD & 31 & 46 & 0.403 & \\
CD & 49 & 32 & 0.605 & \\
$\begin{array}{l}\text { Postnatally } \\
\text { diagnosed OI }\end{array}$ & & & & 0.475 \\
$\quad$ VD & 69 & 116 & 0.373 & \\
CD & 38 & 53 & 0.418 & \\
\hline
\end{tabular}

The increased fracture rate with cesarean delivery (CD) observed only in those with antenatal and not postnatal diagnosis of osteogenesis imperfecta (OI) suggests that this is due to ascertainment bias and that $C D$ is not the driver behind the increased fractures.

aDenotes $\alpha$ value for comparison between vaginal delivery (VD) and CD within each subgroup.

The median birth weights were significantly different between the OI subtypes delivered at term gestation (37-42 weeks); those with OI type I had the highest birth weight (type I vs. III, $P<0.001$; type I vs. IV, $P<0.001$; type III vs. IV, $P=$ 0.004 ) (Figure 1). However, such differences were not observed among those who were delivered preterm (before 37 weeks). The median birth lengths were highest among those with OI type I and lowest among those with OI type III, with statistically significant differences between the various subtypes (type I vs. III; type I vs. IV; type III vs. IV, $P<0.001$ ) (Figure 1). Among those delivered preterm, significant differences were observed only between those with OI types I and III $(P=0.005)$.

\section{Frequency of at-birth fractures by OI subtype}

As expected, individuals with type III OI had the highest rate of at-birth fractures (92.6\%), followed by those with type IV (50.7\%) and type I (17.2\%) (Table 2). The odds ratio (OR) for the presence of at-birth fracture in OI type III was 60.5 when compared with OI type I (95\% CI 25.9-140.9; $P<0.001)$ and 12.2 when compared with OI type IV (95\% CI 5.27-28.3.4; $P<$ $0.001)$. Subjects with OI type IV were five times more likely to have at-birth fractures compared with those with OI type I (OR 4.95; 95\% CI 3.06-8.04; $P<0.001)$. These results are consistent with the well-known clinical severity of the subtypes.

\section{At-birth fracture rates based on delivery method}

To determine whether $\mathrm{CD}$ was a safer mode of delivery for those with OI, we assessed the at-birth fracture rates within each subtype and found that they did not differ based on whether the delivery was by the vaginal route or by $\mathrm{CD}$ (Table 2). The proportion of individuals with at-birth fractures with vaginal delivery and CDS were 0.16 and 0.19 for OI type I $(P=0.561), 0.91$ and 0.94 for OI type III $(P=0.698)$, and 0.55 and 0.46 for OI type IV $(P=0.313)$, respectively. When the data were pooled, however, the fracture rate was higher among those who were delivered by $\mathrm{CD}$ compared with vaginal delivery ( 0.5 vs. 0.39 ; $P=0.014$ ) (Table 2). The OR for fractures with $\mathrm{CD}$ was significantly higher than with vaginal delivery $(1.6 ; 95 \%$ CI $1.10-2.36$; $P=0.014)$. This could imply that $\mathrm{CD}$ either is indeed associated with a higher risk for fracture or this finding is secondary to an ascertainment bias, whereby $\mathrm{CD}$ was chosen as the method of delivery for those who were diagnosed with $\mathrm{OI}$ antenatally. If the latter was the case, such increased risk would be observed only in those individuals diagnosed with OI antenatally and not in those who were diagnosed after birth. In individuals diagnosed antenatally with OI, analysis of pooled data from all subjects showed that the risk for at-birth fracture was elevated with $\mathrm{CD}$ (OR 2.27; 95\% CI 1.20-4.30; $P=0.011$ ); in those diagnosed after birth, however, no such increase was evident (OR 1.20; 95\% CI $0.72-2.01 ; P=0.484$ ) (Table 3 ). When examining only cases with the presence of in utero fracture, there was an increased use of $\mathrm{CD}$ (OR 4.53; 95\% CI 2.31-8.90; $P<0.001)$. These suggest that $\mathrm{CD}$ was likely not the driver behind increased at-birth fracture rates, but rather the delivery method chosen for those who 
Table 4 Subset of patients with fractures presumably sustained during delivery

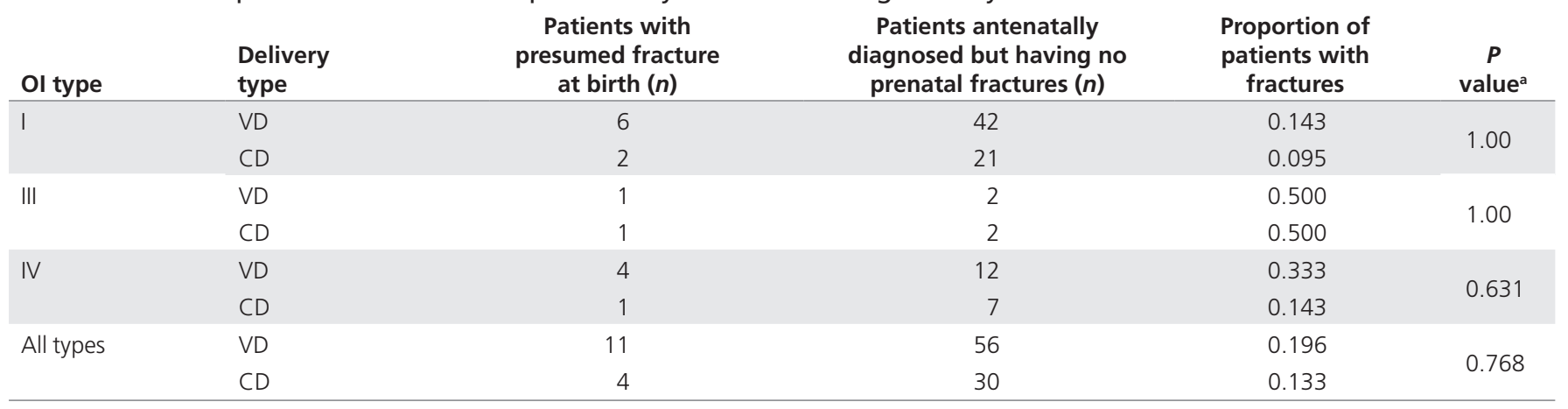

Denotes $\alpha$ value for comparison between vaginal delivery (VD) and cesarean delivery (CD) within each osteogenesis imperfecta (OI) subtype.

had in utero fractures, and thus at-birth fracture. Further analyses with logistic modeling showed that the mode of delivery had no effect on the fracture rates at birth when accounting for the type of OI and the presence of in utero fractures.

The presence or absence of fracture at birth as a categorical variable does not address the question of whether any fractures were sustained during the process of delivery. The limitations in the timing of the ultrasound examination and antenatal quantification of the fractures make this a difficult question to address. Within the limitations of the data set, however, we attempted to address this by analyzing the subgroup of subjects who were antenatally diagnosed with OI but were not known to have any antenatal fractures; birth fractures among these individuals were presumed to occur during delivery. The fracture rate analysis in this subgroup also showed no difference within each subtype, nor in the pooled data based on whether delivery was by the vaginal route or $\mathrm{CD}$ ( $P=1.00$ for type I; $P=1.00$ for type III; $P=0.631$ for type IV; $P=0.768$ for pooled data) (Table 4$)$. We attempted to evaluate the effect of delivery method for fetuses with breech presentation separate from vertex presentation and did not identify significant differences for at-birth fracture rate (breech presentation $P=0.334$; vertex presentation $P=0.496$ ).

A unique scenario is when a mother affected with $\mathrm{OI}$ is pregnant with a fetus also affected with OI. In the LCRC data set, 45 such women ( 36 with OI type I and 9 with OI type IV) were identified. All women with type IV OI delivered by CD. Among the women with OI type I, 18 delivered by $\mathrm{CD}$, whereas 18 delivered by the vaginal route. The at-birth fracture rate among the newborns was not significantly different between vaginal delivery and $\mathrm{CD}$ ( 0.27 vs. 0.25$)$, even in this subcategory of subjects. These results collectively show that $\mathrm{CD}$ is not associated with a decrease in fracture rates at birth.

\section{Factors influencing the choice of delivery method}

The decision to choose a delivery method is dependent on patient and physician preferences in addition to multiple variables specific for OI. We therefore looked for factors that correlated with choosing $\mathrm{CD}$ as a delivery method. Antenatal diagnosis of OI correlated with an increased chance of $\mathrm{CD}(\mathrm{OR}$ 2.139; 95\% CI 1.433-3.190; $P<0.001)$. To further clarify potential correlates, we used backward-elimination logistic regression model building to examine correlations between mode of delivery and three factors that clinicians often consider while deciding the delivery method, namely, presentation at birth, maternal history of OI, and presence of in utero fractures. There was an improvement in the fit of the model when mode of delivery was correlated with all three of these variables $(P<0.001)$. Interactions between these variables did not improve the model, suggesting that presentation at birth, maternal history of OI, and presence of in utero fractures independently and individually influence the decision to perform a CD.

\section{Factors affecting at-birth fracture rate}

The severity of the type of OI was the predominant determinant of the fracture rates at birth. Using a backward-elimination strategy, we assessed the correlation between at-birth fractures and many covariates, and we found that in utero fracture, OI subtype, and birth weight independently correlated $(P<0.001)$ with fracture. Higher birth weight conferred a higher risk for fracture. Overall, though, there was a trend toward higher fracture risk with vaginal delivery compared with $\mathrm{CD}$ with increasing birth weight, but these results were statistically insignificant $(P=0.092)$. We examined interaction terms and included an interaction of antenatal diagnosis with delivery type, but this did not improve the model $(P=0.145)$.

\section{DISCUSSION}

Antenatal suspicion for a skeletal dysplasia such as OI raises many clinically relevant issues, including confirming the diagnosis, assessing the severity of the condition and possible lethality, evaluating the risks posed to the mother, and planning for a delivery that minimizes the risk for fracture. While some studies have addressed the diagnosis and assessment of severity during the antenatal period, no systematic studies provide guidance on further management of pregnancy or delivery. ${ }^{13-18}$ Using systematically collected data from a large cohort of subjects with nonlethal type I collagen-related OI, we tried to address issues often encountered in clinical practice, including the implications of OI on fetal presentation and growth parameters, the effect of mode of delivery on at-birth fracture rates, characteristics that confer a higher risk for fractures, and factors influencing the choice of delivery method. 
The presentation of the fetus during delivery correlates with the severity of OI; nearly $40 \%$ with OI type III have a breech presentation. Overall, irrespective of the type of OI, the incidence of breech presentation was significantly higher than among the general population. While nearly a third of fetuses could be in the breech presentation during second trimester, most of these rotate, so that the overall incidence of breech among the general population is less than $5 \% .{ }^{19-21}$ There is an increased incidence of breech presentation among fetuses with chromosomal aneuploidy; disorders affecting neuromuscular function, such as Prader-Willi syndrome; Zellweger syndrome; spinal muscular atrophy; and developmental abnormalities such as meningomyelocele. ${ }^{20}$ The reasons for increased breech presentation in OI may be the increased head-to-body ratio, longbone fractures, and other bony deformities that impede fetal rotation. Our results are consistent with observations made by Cubert and colleagues ${ }^{11}$ in a smaller cohort of subjects with OI. The incidence of preterm delivery in OI was not significantly increased compared with data from the general population, and the majority of fetuses were delivered at term gestation. ${ }^{22}$ The birth weight and length were smallest among fetuses with OI type III followed by those with OI types IV and I. These data suggest that growth restriction correlates with the type of OI. Similar differences were not discernible in those delivered before 37 weeks' gestation, probably because the varying gestational ages at delivery contributed to a large dispersion around the measure of central tendency.

The in utero and at-birth fracture rates were highest among fetuses with OI type III, and those with this severe form of OI were $\sim 60$ times more likely to have an at-birth fracture than those with type I OI. The subtype of OI was the single most important factor that determined fracture rates. Some have advocated that CD be preferentially performed with antenatal diagnosis of OI because this method is quicker and is supposed to provide more control over the delivery. ${ }^{8-10}$ However, such recommendations do not account for the fact that CD involves significant traction forces on the fetal limbs through a relatively small incision in the uterine wall, compared with the relatively uniform pressure exerted by the muscular birth canal during vaginal delivery. To date, there has been no evidence to show that CD leads to a decrease in fracture rates at birth or increases survival during the perinatal period. Our results clearly show that $\mathrm{CD}$ is not associated with a decrease in the at-birth fracture rates when accounting for the type of OI. About $90 \%$ of subjects with OI type III, 50\% with OI type IV, and $\sim 17 \%$ with OI type I have fractures at birth irrespective of the mode of delivery. Interestingly, when data were analyzed collectively without categorizing subjects by OI subtype, CD seemed to be associated with an increase in the risk for fracture. However, this was from an ascertainment bias as a result of CD being the "chosen" method of delivery for individuals who were antenatally diagnosed with OI. The large size of the cohort allowed us to further investigate potential correlates that had an outcome on fracture rates. We found that only birth weight independently correlated with fracture, and those with higher birth weight were at an increased risk. Fetal macrosomia, in general, has been associated with increased trauma during delivery, and macrosomic fetuses (weight $>4 \mathrm{~kg}$ ) have higher rates of clavicular and shoulder fractures. ${ }^{23-25}$ There was a trend toward higher fracture risk with increasing birth weight with vaginal delivery, though these results were statistically insignificant. Given the risks for shoulder dystocia with higher birth weights, CD could be potentially considered in OI in this specific scenario.

The question of which method results in fewer fractures during the process of delivery is a difficult one to answer. The practicality and limitations of sensitivity and expertise in quantifying antenatal fractures preclude an accurate differentiation between antenatal fractures and those that occur during the intrapartum period. We presumed that the at-birth fractures in the subset of subjects who were antenatally diagnosed with OI but were not known to have any antenatal fractures occurred during delivery, and we analyzed the fracture rate based on delivery method. This analysis also showed that fracture rates were not different between $\mathrm{CD}$ and vaginal delivery.

A situation that is infrequently encountered is that of a woman with OI who is pregnant with a fetus affected with OI. Women with types III and IV OI can have pelvic and long-bone deformities, and often CD would be required for delivery of the fetus because of cephalopelvic disproportion or concerns of maternal fractures during delivery., ${ }^{9,26,27}$ In fact, there have been anecdotal reports wherein $\mathrm{CD}$ had to be performed to deliver even a nonviable fetus. ${ }^{28}$ In our cohort, all nine women with OI type IV delivered by CD. However, in the OI type I group, wherein equal numbers of individuals were delivered by $\mathrm{CD}$ and the vaginal routes, the fracture rate did not differ based on mode of delivery. Data regarding maternal fractures during delivery was not available.

The choice of delivery method was dependent on many factors, including antenatal diagnosis, presence of in utero fractures, breech presentation, and maternal history of a moderate form of OI. These results are discordant from the results of Cubert et al., ${ }^{11}$ who noted that fetal malpresentation was the main reason behind $\mathrm{CD}$ in their cohort. The discrepancy in the findings is explicable by the smaller sample size and the unavailability of pregnancy-related data in their study.

The results from our study have to be interpreted within the context of the limitations of the data. All of the data points of interest, including antenatal diagnosis, mode of delivery, and the presence or absence of fractures at birth, were self-reported. These data could not be confirmed by a review of medical records. Any such data set is bound to have some element of recall bias, especially in subjects who are older. The at-birth fracture rate is categorical data and cannot determine whether there was a difference in the number of fractures between the two delivery methods. In addition, clinically relevant questions, such was whether a trial of labor in those who were delivered by $\mathrm{CD}$ was associated with increased fracture risk, or whether the location of fracture was significantly different based on delivery type, cannot be answered from the current data. We were also unable to account for complications that arise at the time 
of delivery, which may be associated with fracture risk, such as protracted labor or use of instrumentation in a vaginal delivery. In spite of these limitations, our analyses of the largest cohort of subjects with OI have many strengths as well. The systematic collection of specific data points as defined by preset conditions and monitoring by a central committee ensures uniformity and high quality data. The large sample size allows for statisti$\mathrm{cal}$ analysis with sufficient power to answer clinically relevant questions and can inform clinical decisions. The lessons learned from this experience are being used to prospectively collect data for a natural history study conducted by the Brittle Bone Disease Consortium, a Rare Disease Clinical Research Network of the National Institutes of Health. These efforts ultimately aim to develop guidelines for the management of pregnancy in OI and underscore the utility of collaborative research networks in improving the clinical care of patients with rare disorders.

In summary, our study, the largest to assess the factors that determine fracture rate at birth in OI, shows that CD is not associated with decreased risk for at-birth fractures. Thus, CD should be performed only for other maternal or fetal indications, not for sole purpose of fracture prevention in OI.

\section{ACKNOWLEDGMENTS}

This work was supported by the Brittle Bone Disease Consortium (1U54AR068069-0), a part of the National Center for Advancing Translational Sciences (NCATS) Rare Disease Clinical Research Network, and is funded through a collaboration between the Office of Rare Diseases Research, NCATS, the National Institute of Arthritis and Musculoskeletal and Skin Diseases (NIAMS), and the National Institute of Dental and Craniofacial Research. The content is solely the responsibility of the authors and does not necessarily represent the official views of the National Institutes of Health $(\mathrm{NIH})$. The Brittle Bone Disease Consortium is also supported by the Osteogenesis Imperfecta Foundation. The project was also supported by Baylor College of Medicine Intellectual and Developmental Disabilities Research Center (grant 1 U54 HD083092) from the Eunice Kennedy Shriver National Institute of Child Health \& Human Development. S.B. was the recipient of the Texas Department of Health Services Summer Scholarship program for 2014. M.J. was funded by an NIH training grant (T32GM07526). S.C.N. is a recipient of the Doris Duke Charitable Foundation Clinical Scientist Development Award, and this work was supported by the Doris Duke Charitable Foundation (grant 2013095). The authors thank the members of the Brittle Bone Disease Consortium: David Eyre, Gerald Harris, Eric Orwoll, Cathleen Raggio, Laura Tosi, and Anne Tsai. They also recognize the contributions of clinical research teams at the respective sites: Mary Mullins, Alyssa Tran, Susan Carter (Baylor College of Medicine and Texas Children's Hospital); Vonda Vensel, Jill Christie, and Abigail Hata (Oregon Health \& Science University); Michaela Durigova (Shriners Hospital Montreal); and Lauren Davey (A.I. duPont Hospital for Children).

\section{DISCLOSURE}

The authors declare no conflict of interest.

\section{REFERENCES}

1. Rauch F, Glorieux FH. Osteogenesis imperfecta. Lancet 2004;363:1377-1385.

2. Marini JC, Blissett AR. New genes in bone development: what's new in osteogenesis imperfecta. J Clin Endocrinol Metab 2013;98:3095-3103.

3. Patel RM, Nagamani SC, Cuthbertson D et al. A cross-sectional multicenter study of osteogenesis imperfecta in North America - results from the linked clinical research centers. Clin Genet 2015;87:133-140.

4. Sillence DO, Senn A, Danks DM. Genetic heterogeneity in osteogenesis imperfecta. J Med Genet 1979;16:101-116.

5. Aerts M, Van Holsbeke C, de Ravel T, Devlieger R. Prenatal diagnosis of type Il osteogenesis imperfecta, describing a new mutation in the COL1A1 gene. Prenat Diagn 2006;26:394.

6. Byers PH, Krakow D, Nunes ME, Pepin M; American college of medical genetics. Genetic evaluation of suspected osteogenesis imperfecta (OI). Genet Med 2006:8:383-388.

7. Steiner RD, Adsit J and Basel D. COL1A1/2-related osteogenesis imperfecta. In: Pagon RA et al. (eds.) GeneReviews. University of Washington: Seattle, WA, 1993.

8. Marini JC. Osteogenesis imperfecta: comprehensive management. Adv Pediatr 1988;35:391-426.

9. Roberts JM, Solomons CC. Management of pregnancy in osteogenesis imperfecta: new perspectives. Obstet Gynecol 1975;45:168-170.

10. Sharma A, George L, Erskin K. Osteogenesis imperfecta in pregnancy: two case reports and review of literature. Obstet Gynecol Surv 2001;56:563-566.

11. Cubert R, Cheng EY, Mack S, Pepin MG, Byers PH. Osteogenesis imperfecta: mode of delivery and neonatal outcome. Obstet Gynecol 2001;97:66-69.

12. Lindahl K, Åström E, Rubin CJ, et al. Genetic epidemiology, prevalence, and genotype-phenotype correlations in the Swedish population with osteogenesis imperfecta. Eur J Hum Genet 2015;23:1112.

13. Krakow D, Lachman RS, Rimoin DL. Guidelines for the prenatal diagnosis of fetal skeletal dysplasias. Genet Med 2009;11:127-133.

14. Barkova E, Mohan U, Chitayat D, et al. Fetal skeletal dysplasias in a tertiary care center: radiology, pathology, and molecular analysis of 112 cases. Clin Genet 2015;87:330-337.

15. Krakow D, Alanay $Y$, Rimoin LP, et al. Evaluation of prenatal-onset osteochondrodysplasias by ultrasonography: a retrospective and prospective analysis. Am J Med Genet A 2008;146A:1917-1924.

16. Pepin M, Atkinson M, Starman BJ, Byers PH. Strategies and outcomes of prenatal diagnosis for osteogenesis imperfecta: a review of biochemical and molecular studies completed in 129 pregnancies. Prenat Diagn 1997;17: 559-570.

17. Schramm T, Gloning KP, Minderer S, et al. Prenatal sonographic diagnosis of skeletal dysplasias. Ultrasound Obstet Gynecol 2009;34:160-170.

18. Wu Q, Wang W, Cao L, Sun L, Xu Y, Zhong X. Diagnosis of fetal osteogenesis imperfecta by multidisciplinary assessment: a retrospective study of 10 cases. Fetal Pediatr Pathol 2015;34:57-64.

19. Berendes HW, Weiss W, Deutschberger J, Jackson E. Factors associated with breech delivery. Am J Public Health Nations Health 1965;55:708-719.

20. Braun FH, Jones KL, Smith DW. Breech presentation as an indicator of fetal abnormality. J Pediatr 1975;86:419-421.

21. Vartan CK. The behaviour of the foetus in utero with special reference to the incidence of breech presentation at term. J Obstet Gynaecol Br Emp 1945;52:417-434.

22. Beck S, Wojdyla D, Say L, et al. The worldwide incidence of preterm birth: a systematic review of maternal mortality and morbidity. Bull World Health Organ 2010;88:31-38.

23. Boyd ME, Usher RH, McLean FH. Fetal macrosomia: prediction, risks, proposed management. Obstet Gynecol 1983;61:715-722.

24. Lam MH, Wong GY, Lao TT. Reappraisal of neonatal clavicular fracture: relationship between infant size and neonatal morbidity. Obstet Gynecol 2002;100:115-119.

25. Wollschlaeger K, Nieder J, Köppe I, Härtlein K. A study of fetal macrosomia. Arch Gynecol Obstet 1999;263:51-55.

26. Krishnamoorthy U, Vausse S, Donnai P. Management of pregnancy complicated by maternal osteogenesis imperfecta. Report of a case with uterine rupture. J Obstet Gynaecol 2002;22:316.

27. Vogel TM, Ratner EF, Thomas RC Jr, Chitkara U. Pregnancy complicated by severe osteogenesis imperfecta: a report of two cases. Anesth Analg 2002;94:1315-1317.

28. Feng ZY, Chen Q, Shi CY, Wen HW, Ma K, Yang HX. A type IV osteogenesis imperfecta family and pregnancy: a case report and literature review. Chin Med $J$ (Engl) 2012;125:1358-1360. 